\title{
The Method of Lines and Adomian Decomposition for Obtaining Solitary Wave Solutions of the KdV Equation
}

\author{
Mohamed M. Mousa ${ }^{1} \&$ Mohamed Reda ${ }^{1}$ \\ ${ }^{1}$ Department of Basic Science, Benha Faculty of Engineering, Benha University, Benha, Egypt \\ Correspondence: Mohamed M. Mousa, Department of Basic Science, Benha Faculty of Engineering, Benha \\ University, Benha 13512, Egypt. E-mail: mohamed.youssef@bhit.bu.edu.eg
}

Received: August 23, 2012 Accepted: February 21, 2013 Online Published: April 1, 2013

doi:10.5539/apr.v5n3p43 URL: http://dx.doi.org/10.5539/apr.v5n3p43

\begin{abstract}
The method of lines (MOL) and Adomian decomposition method (ADM) are presented for obtaining solitary wave solutions of the korteweg-de Varies equation $(\mathrm{KdV})$. The numerical results of the MOL are compared with the analytical results of the ADM. The method of lines gives accurate results over the Adomian method. In order to show the reliability of the considered methods we have compared the obtained solutions with the exact ones. The results reveal that the method of lines is more effective and convenient than the Adomian decomposition method for solving such type of partial differential equations.
\end{abstract}

Keywords: KdV equation, the method of lines, Adomian decomposition method, finite difference scheme, Runge-Kutta method

\section{Introduction}

Nonlinear wave equations, such as the $\mathrm{KdV}$ equation, in particular have provided several examples of solutions that are strikingly different from those obtained for linear wave propagation. The ones that have attracted the attention are the solitary waves, or solitons. The term soliton was used by Zabusky and Kruskal to emphasize the localized nature of these solutions and retention of their form after interactions with other solitons (Zabusky \& Kruskal, 1965; Ozis \& Ozer, 2006; Hirota, 1973a, 1973b). The Solitary wave is a traveling wave of the form $u(x$, $\mathrm{t})=f(x, t)$ for some smooth function $f$ that decays rapidly at infinity. Several properties are generally found for this type of solution to nonlinear equations: (i) the form is localized in the sense that the solitary wave becomes constant (possibly zero) at infinity, so the derivatives of the solution vanish at infinity (ii) a single soliton propagates without change in form and, most strikingly (iii) solitons interacting with other solitons retain their original form after interaction. For these reasons, solitons are said to have particle-like properties. For more details about the formulation of KdV equation, see (Korteweg \& de Vries, 1895; Luwai, 2009; Khattak \& Siraj-ul-Islam, 2008; Mousa, Ragab Shahwar, \& Mostafa, 2006; Kening, Ponce, \& Vega, 1997; Lax, 1978). The models of $\mathrm{KdV}$ equation is given by

$$
u_{t}+\varepsilon u u_{x}+u u_{x x x}=0
$$

$u(x, 0)=f(x)$, where $\varepsilon$ and $\mu$ are constants and Equation (1) is the pioneering equation that gives rise to solitary wave solutions. The method of lines and Adomian decomposition method are used to study the KdV equation and to show that these methods are effective in solving a large number of linear or nonlinear partial differential equations.

The KdV Equation (1) describes motions of long wave in shallow water under gravity and in a one- dimensional nonlinear lattice (Gardner, L. R. T., \& Gardner, G. A., 1992; Debnath, 1994). This particular form (1) is of special interest (Debnath, 1998; Gardner, L. R. T., \& Gardner, G. A., 1990) because it admits inverse scattering formalism. The considered is an important mathematical model with wide applications in quantum mechanics and nonlinear optic .Typical examples are used in various fields such as solid state physics, plasma physics, fluid physics and quantum field theory.

The Method of Lines is a partial discretization technique. This numerical method forms a versatile tool for the analysis of optical and microwave waveguides (Wazwaz, 2001; Baker, 1996, 1975; Schiesser, 1991). Due to its 
partial discretization approach, the computational effort is much less than other full discretization methods applied to the same problem. According to the MOL, the equations are discretized only as far as necessary and all other calculations are done analytically or numerically. As interface conditions can be employed in this method, discontinuous filed curves can be described accurately. This leads to the popularity of this method for solving more complicated problems.

Adomian decomposition method is the only decomposition method for solving differential equations of various types and can solve various equations without discretization or invalidation. This method, as well as Adomian polynomials of various orders, is used in solving linear and nonlinear derivable equations, without linearization of nonlinear equations of various orders (Mehdi, 2004; Wazwaz, 2001). In general, the ADM is believed to be an easy and convenient tool with wide applicability. However, some discretion is recommended for its use as there are certain conditions which limit the quality of the solution generated.

There exist some techniques to accelerate the convergence of a given series solution obtained by ADM. Among them, the so-called Padé technique is widely applied. It is known that Padé approximations (PA) have the advantage of manipulating the polynomial and series approximation into a rational function of polynomials (Baker, 1975, 1996). This rational function provides us with more information about the mathematical manner of the solution.

\section{The Method of Lines}

The method of lines (Schiesser, 1991) is a well established numerical technique (or rather a semi analytical method) for the analysis of transmission lines and waveguide (Yang \& Pregla, 1995; Pregla \& Yang, 1993; Pregla \& Ahlers, 1993; Rogge \& Pregla, 1991). The method of lines is regarded as a special finite difference method but more effective with respect to accuracy and computational time than the regular finite difference methods. It basically involves discretising a given differential equation in one or two dimensions while using analytical solution in the remaining direction. The MOL has the merits of both the finite difference method and analytical method, it does not yield spurious modes nor have the problem of relative convergence. The MOL is generally recognized as a comprehensive and powerful approach to the numerical solution of time-dependent partial differential equations (PDEs). This method usually proceeds in two separate steps: first, approximating the spatial derivatives. Second, the resulting system of semi discrete (discrete in space-continuous in time) ordinary differential equations (ODEs) is integrated in time. The essence of the method of lines is a way of approximating PDEs by ODEs. Obviously, an advantage of the MOL is that one can use all kinds of ODE solvers and techniques to solve the semi-discrete ODEs directly.

\section{Solving the KdV Equation Using the MOL}

Consider KdV Equation (1) with initial condition

$$
u(x, 0)=\frac{\alpha}{2}\left(\operatorname{sech}^{2}\left(\frac{\sqrt{\alpha}}{2} x\right)\right), a \leq x \leq b
$$

and the boundary conditions

$$
u_{(a, t)}=0, u_{(b, t)}=0, t>0
$$

The solution of the method of lines using fourth order finite difference scheme for both $u_{x}$ and $u_{x x x}$ is denoted by MOLI, however the solution using a second order finite difference scheme for both $u_{x}$ and $u_{x x x}$ is denoted by MOLII.

The derivative $u_{x}$ in KdV Equation (1) is computed by finite differences scheme in two ways

1) three-point centered approximations (second order finite difference scheme)

$$
u_{x}=\frac{u_{i+1}-u_{i-1}}{2 h}+O(\mathrm{~h})^{2}
$$

2) seven-point centered approximations (fourth order finite difference scheme)

$$
u_{x}=\frac{u_{i-2}-8 u_{i-1}+8 u_{i+1}-u_{i+2}}{12 h}+O(\mathrm{~h})^{4}
$$

The derivative $u_{x x x}$ in KdV Equation (1) is computed by finite differences in two ways 
1) five-point centered approximations (second order finite difference scheme)

$$
u_{x x x}=\frac{-u_{i-2}+2 u_{i-1}+2 u_{i+1}+u_{i+2}}{2 h^{3}}+O(\mathrm{~h})^{2}
$$

2) seven-point centered approximations (fourth order finite difference scheme)

$$
u_{x x x}=\frac{u_{i-3}-8 u_{i-2}+13 u_{i-1}-13 u_{i+1}+8 u_{i+2}-u_{i+3}}{8 h^{3}}+O(\mathrm{~h})^{4}
$$

Applying the above finite difference schemes to Equation (1) yields a system of ordinary differential equations for the unknown $u_{i}$ as functions in $\mathrm{t}$ as follows:

$$
\frac{d u_{i}(t)}{d t}=f\left(u_{i}\right), i=1,2,3, \ldots \ldots \ldots . . .1
$$

Using the fourth order finite difference scheme both $u_{x}$ and $u_{x x x}$ (MOLI), we have

$$
\begin{aligned}
& \frac{d u_{i}(t)}{d t}=\frac{-u\left(u_{i-3}(t)-8 u_{i-2}(t)+13 u_{i-1}(t)-13 u_{i+1}(t)+8 u_{i+2}(t)-u_{i+3}(t)\right)}{8 h^{3}} \\
& -\varepsilon u_{i}(t) \frac{u_{i-2}(t)-8 u_{i-1}(t)+8 u_{i+1}(t)-u_{i+2}(t)}{12 h}, i=1,2,3, \ldots \ldots . . N-1
\end{aligned}
$$

Using the second order finite difference scheme both $u_{x}$ and $u_{x x x}$ (MOLII), we have

$$
\begin{gathered}
\frac{d u_{i}(t)}{d t}=\frac{-\mu\left(-u_{i-2}(t)+2 u_{i-1}(t)+2 u_{i+1}(t)+8 u_{i+2}(t)\right)}{2 h^{3}} \\
-\varepsilon u_{i}(t) \frac{u_{i+1}(t)-u_{i-1}(t)}{2 h}, i=1,2,3, \ldots \ldots \ldots . . N-1
\end{gathered}
$$

Then, any popular ODE solvers has to be used for solving (4), we will use classical four order Runge-Kutta scheme (RK4)

$$
\begin{gathered}
U^{n+1}=U^{n}+\frac{\Delta t\left(K_{1}+2 K_{2}+2 K_{3}+K_{4}\right)}{6}, \quad K_{1}=F\left(U^{n}\right) \\
K_{2}=F\left(U^{n}+\frac{\Delta t}{2} K_{1}\right), K_{3}=F\left(U^{n}+\frac{\Delta t}{2} K_{2}\right), K_{4}=F\left(U^{n}+\Delta t^{*} K_{3}\right)
\end{gathered}
$$

We will present two numerical examples. One simulates the propagation of a single soliton and the other simulates the interaction of two solitons as famous KdV equation solutions.

Example 1. Propagation of a single soliton (see Drazin, 1989). We study a single soliton of Equation (1) with $\varepsilon=6$ and $\mu=1$. The initial condition and the boundary conditions are shown in (2) and (3)

The exact solution of this problem is given by

$$
u(x, t)=\frac{\alpha}{2}\left(\operatorname{sech}^{2}\left(\frac{\sqrt{\alpha}}{2}(x-\alpha t)\right)\right)
$$

The computational domain is $[-10,20] *[0,5]$. The computational results are listed in Tables 1 and 2 . We plot the profiles of the single soliton at $\alpha=1, \mathrm{t}=0,1,2,3,4,5$ in Figure 1 . 

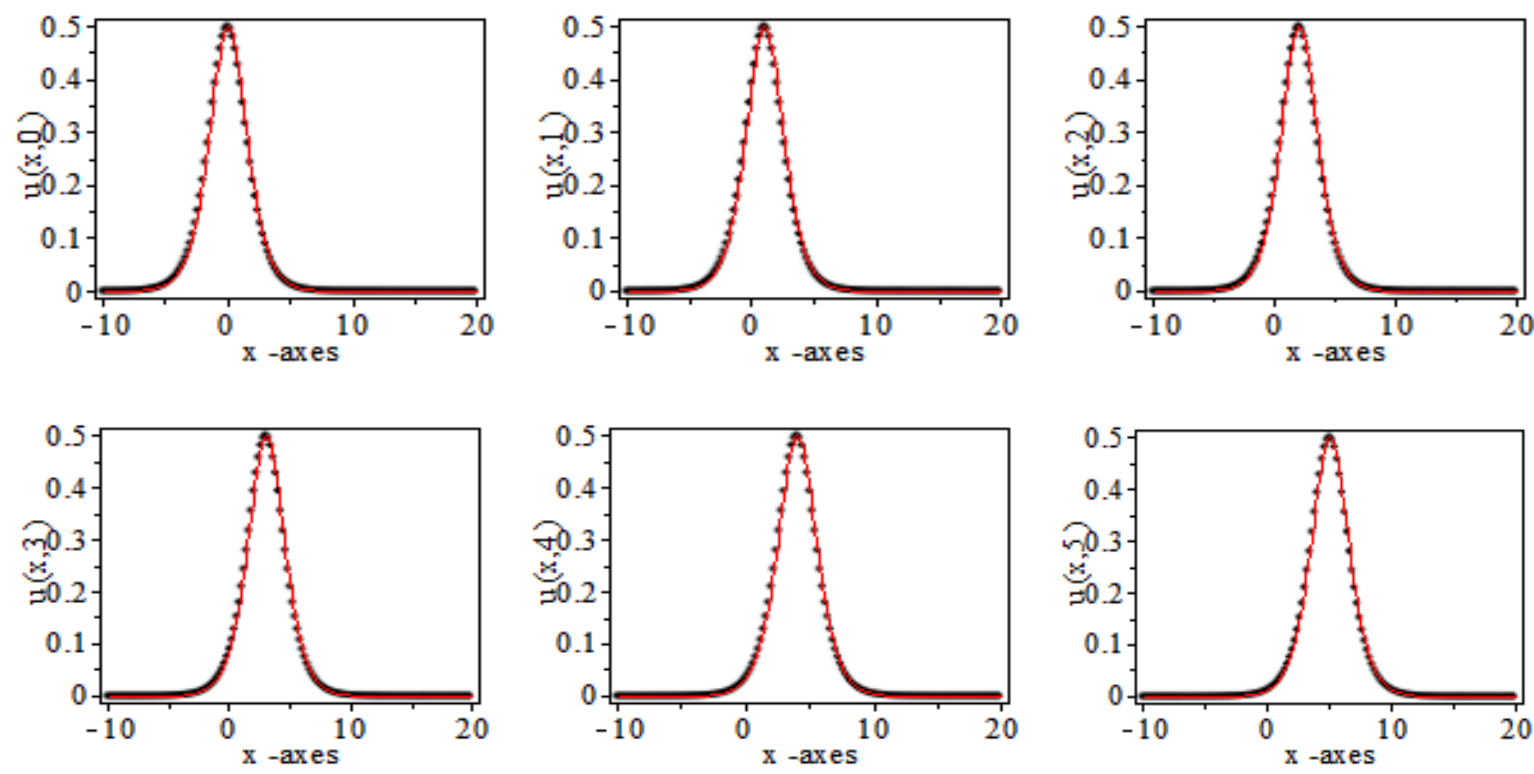

Figure 1. Comparison of MOL I (dotted line) and exact (solid line) solutions corresponding to Example 1 at $\mathrm{N}=200, \alpha=1, \mathrm{t}=0,1,2,3,4,5$
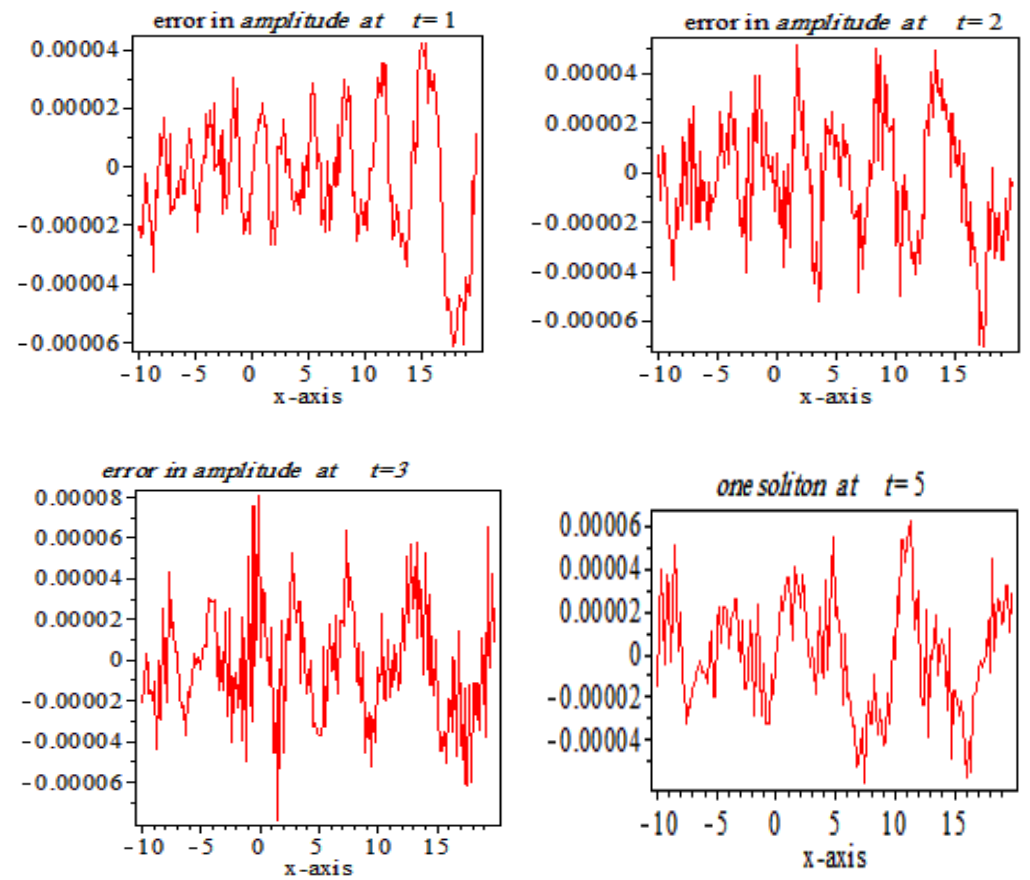

Figure 2. The error between the exact solution $\mathrm{u}(\mathrm{x}, \mathrm{t})$ and the (MOL I) solution for Example 1at $\mathrm{N}=200, \alpha=1$ and $\mathrm{t}=1,2,3,5$ 
The solution profiles of Example 1 using (MOL I) at $\alpha=4$ and $t=0,1,4$ in next figures.
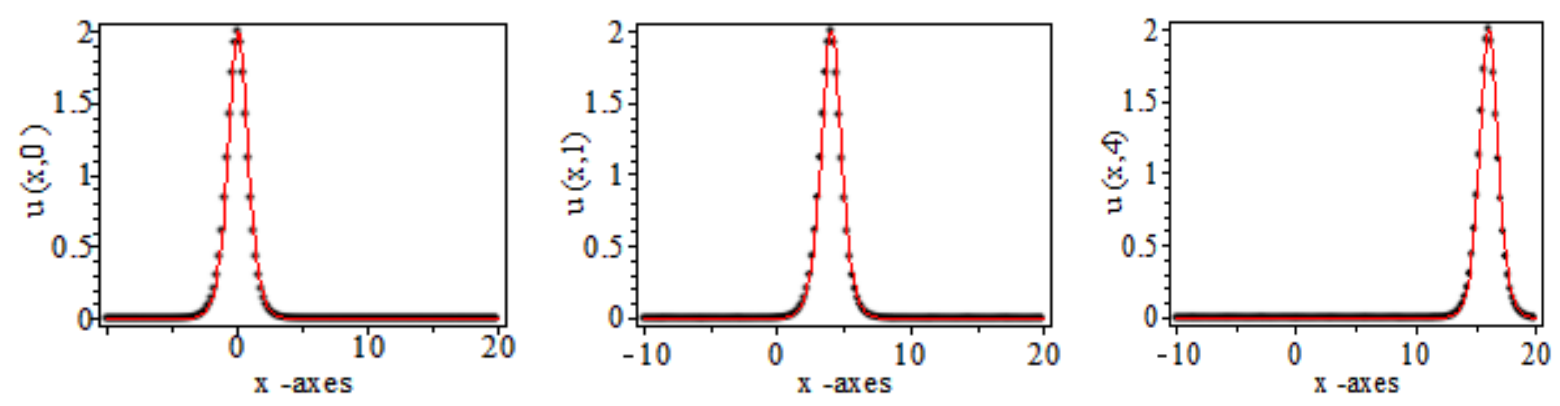

Figure 3. Comparison of MOL I (dotted line) and exact (solid line) solutions corresponding to Example 1 at $\mathrm{N}$ $=200, \alpha=4$ and $\mathrm{t}=0,1,4$
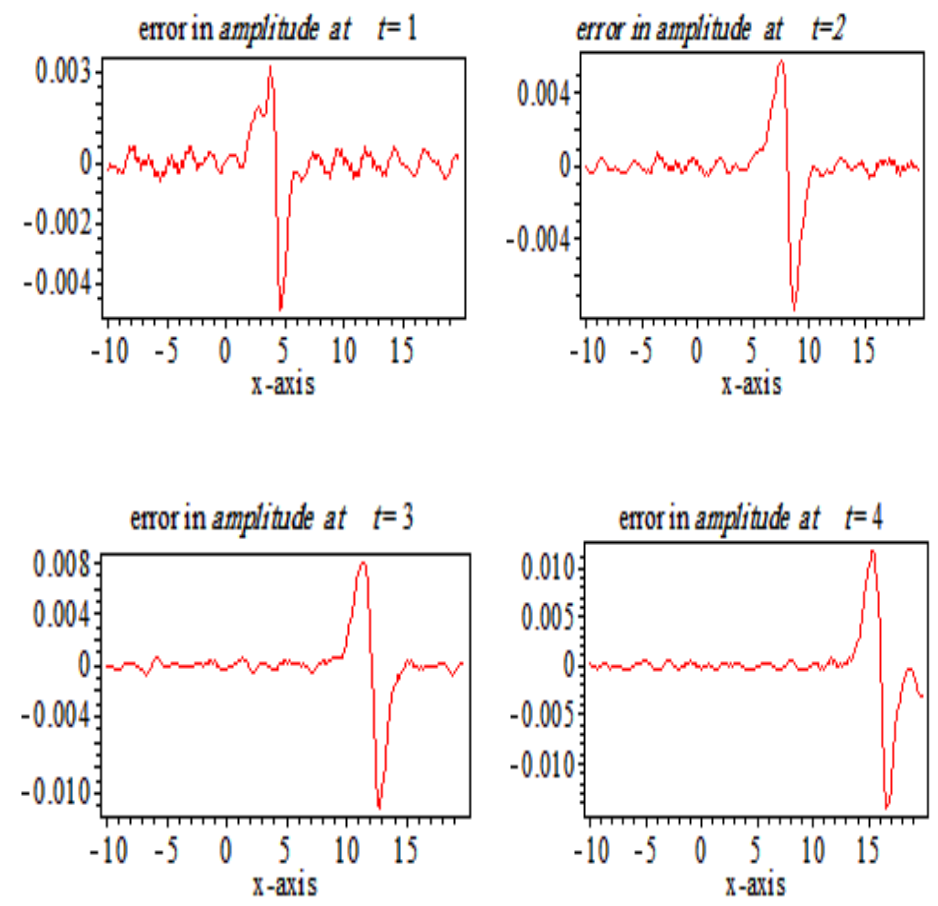

Figure 4. The error between the exact solution $\mathrm{u}(\mathrm{x}, \mathrm{t})$ and the (MOL I) solution for Example 1 at $\mathrm{N}=200, \alpha=4$ and $\mathrm{t}=1,2,3,5$

Example 2. The interaction of two solitons (Drazin, 1989).

In this example, we study the interaction of two solitons of Equation (1) with $\varepsilon=6$ and $\mu=1$. The initial condition is

$$
u(x, 0)=\frac{12(3+4 \cosh (2 x)+\cosh (4 x)}{(3 \cosh (x)+\cosh (3 x))^{2}}
$$

The exact solution of Equation (1) subjet to the intial condition (6) is given by 


$$
u(x, t)=\frac{12(3+4 \cosh (2 x-8 t)+\cosh (4 x-64 t)}{(3 \cosh (x-28 t)+\cosh (3 x-36 t))^{2}}
$$

The computational domain is $[-15,15] *[-0.8,0.8]$. The computational results are listed in Tables 3 and 4 . We also plotted the profiles of the interaction of two solitons using (MOL I) at $\mathrm{N}=500$ and $\mathrm{t}$ from -0.8 to 0.8 .
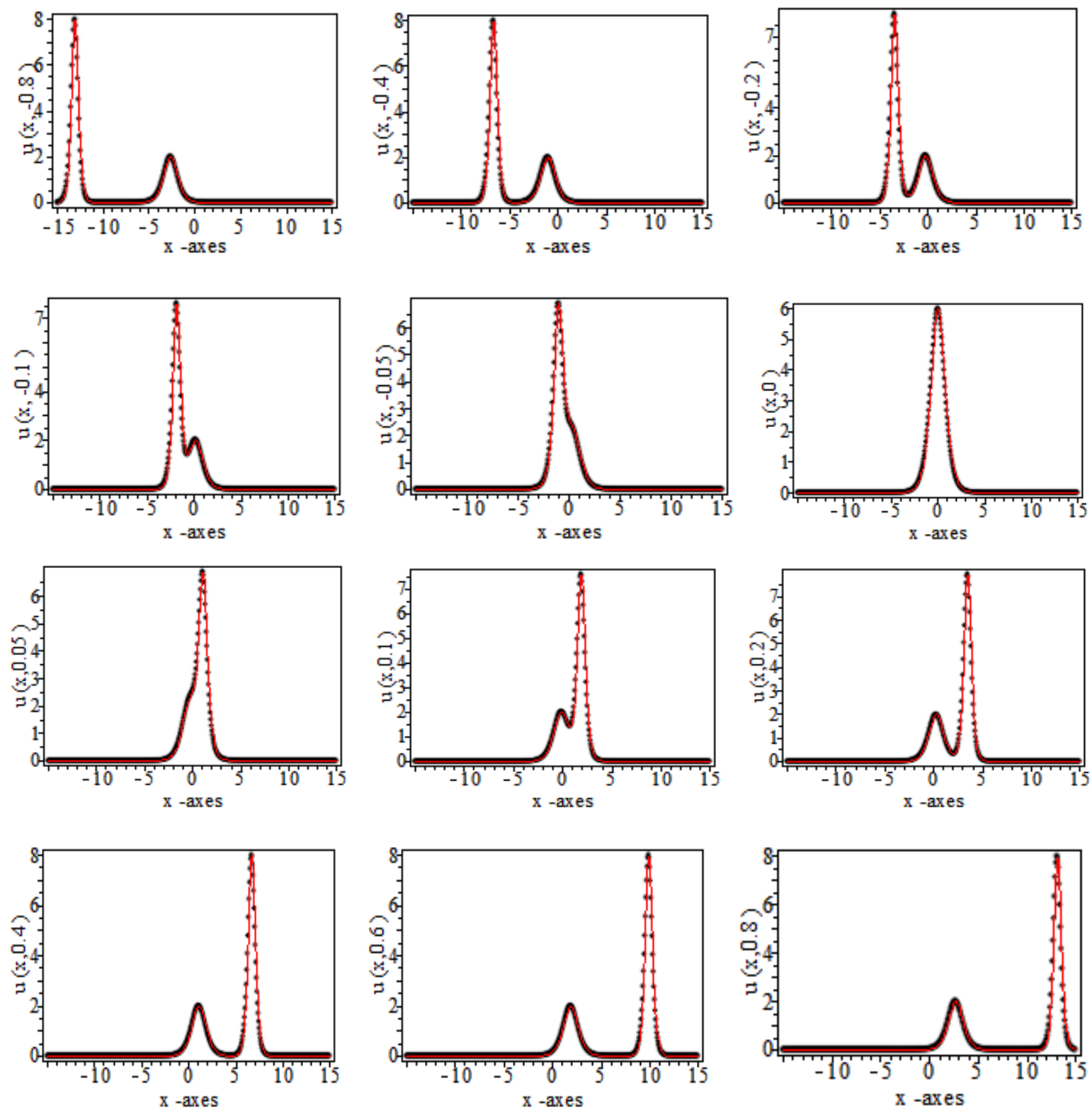

Figure 5. Comparison of MOL I (dotted line) and exact (solid line) solutions corresponding to Example 2 at $\mathrm{N}=500$ and $\mathrm{t}$ from -0.8 to 0.8 

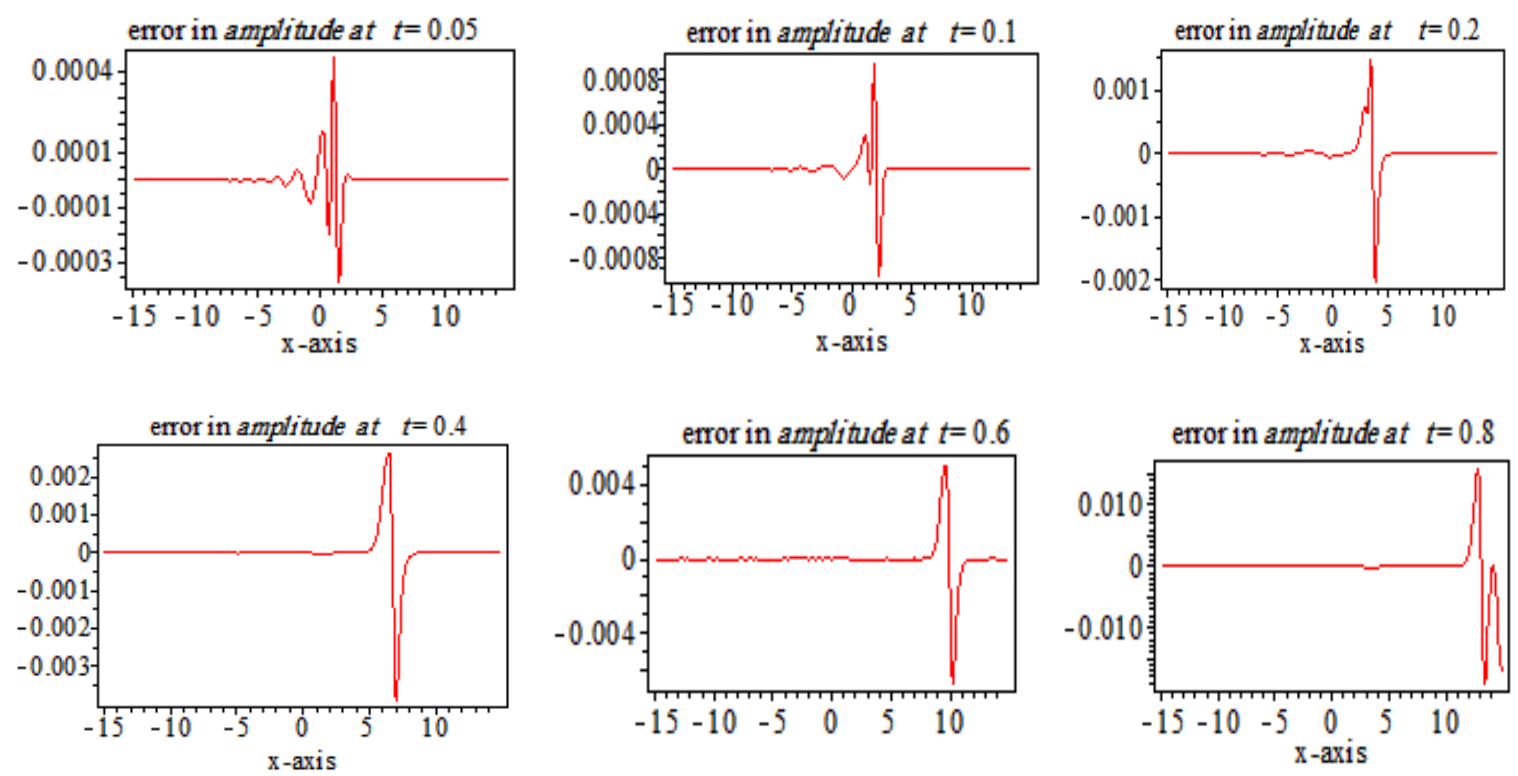

Figure 6. The error between the exact solution $\mathrm{u}(\mathrm{x}, \mathrm{t})$ and the numerical solution for Example 2 using MOL I at $\mathrm{N}=500, \mathrm{t}=0.05,0.1,0.2,0.4,0.6,0.8$

\section{Adomian Decomposition Method}

Following the analysis of Adomian (1994), Equation (1) can be rewritten in an operator form as the following:

$$
L(u)+R(u)+N(u)=g(t)
$$

Where $L=\frac{\partial}{\partial t}$ is the operator of the highest-ordered derivatives with respect to $t$ and $R$ is the remainder of the linear operator. The nonlinear term is represented by $N(u)$. Thus we get

$$
\mathrm{L}(\mathrm{u})=\mathrm{g}(\mathrm{t})-\mathrm{R}(\mathrm{u})-\mathrm{N}(\mathrm{u})
$$

The inverse $L^{-1}$ is assumed an integral operator given by

$$
L^{-1}=\int_{0}^{t}(.) d t
$$

The operating with the operator $L^{-1}$ on both sides of Equation (9) we have

$$
\mathrm{u}=\mathrm{f}_{0}+\mathrm{L}^{-1}(\mathrm{~g}(\mathrm{t})-\mathrm{R}(\mathrm{u})-\mathrm{N}(\mathrm{u}))
$$

Where $f_{0}$ is the solution of homogeneous equation

$$
L(u)=0
$$

The integration constants involved in the solution of homogeneous Equation (12) are to be determined by the initial or boundary condition according as the problem is initial-value problem or boundary-value problem. The Adomian method assumes that the unknown function $\mathrm{u}(\mathrm{x}, \mathrm{t})$ can be expressed by an infinite series of the form

$$
u(x, t)=\sum_{n=0}^{\infty} u_{n}(x, t)
$$

and the nonlinear operator $\mathrm{F}(\mathrm{u})$ can be decomposed by an infinite series of polynomials given by

$$
F(u)=\sum_{n=0}^{\infty} A_{n}
$$

Where $u_{n}(x, t)$ will be determined recurrently, and $A_{n}$ are the so-called polynomials of $u_{0}, u_{1}, u_{2}, \ldots u_{n}$ defined 
by

$$
A_{n}=\frac{1}{n !} \frac{d^{n}}{\mathrm{~d} \lambda^{n}}\left[F\left(\sum_{i=0}^{\infty} \lambda^{i}\right]_{\lambda=0}, n=0,1,2,3, \ldots\right.
$$

It is now well known in the literature that these polynomials can be constructed for all classes of nonlinearity according to algorithms set by Adomian (1994) and Wazwaz (1999).

\section{Solving of KdV Equation Using ADM}

In the following section, we discuss single soliton (Example 1) and two solitons (Example 2) solution of the $\mathrm{KdV}$ equation using ADM. Equation (1) can be written in an operator form:

$$
\mathrm{Lu}=6 \mathrm{u} u_{x}-u_{x x x}
$$

where the differential operator $\mathrm{L}$ is $\mathrm{L}=\frac{\partial}{\partial t}$.

Applying the inverse operator $L^{-1}$ on both sides of (16) And using the decomposition series (13) And (14) yield

$$
\sum_{n=0}^{\infty} u_{n}(x, t)=\frac{\alpha}{2}\left(\operatorname{sech}^{2}\left(\frac{\sqrt{\alpha}}{2} x\right)\right)+L^{-1}\left(-6\left(\sum_{n=0}^{\infty} \mathrm{A}_{n}\right)-\left(\sum_{n=0}^{\infty} u_{n}\right)_{x x x}\right)
$$

Where $A_{n}$ are Adomian polynomials that represent the nonlinear term $u_{x}$ and given by

$$
\begin{gathered}
A_{0}=u_{0 x} u_{0} \\
A_{1}=u_{0 x} u_{1}+u_{1 x} u_{0} \\
A_{2}=u_{0 x} u_{2}+u_{1 x} u_{1}+u_{2 x} u_{0} \\
A_{3}=u_{0 x} u_{3}+u_{1 x} u_{2}+u_{2 x} u_{1}+u_{3 x} u_{0} \\
A_{4}=u_{0 x} u_{4}+u_{1 x} u_{3}+u_{2 x} u_{2}+u_{3 x} u_{1}+u_{4 x} u_{0}
\end{gathered}
$$

Other polynomials can be generated in a like manner. The first few components of $u_{n}(x, t)$ follows as

$$
\begin{gathered}
u_{0}(x)=f(x) \\
u_{1}(x, t)=L^{-1}\left(-6 A_{0}-u_{0 x x x}\right) \\
u_{2}(x, t)=L^{-1}\left(-6 A_{1}-u_{1 x x x}\right) \\
u_{3}(x, t)=L^{-1}\left(-6 A_{2}-u_{2 x x x}\right)
\end{gathered}
$$

The scheme in (18) can easily determine the components $u_{n}(x, t), n \geq 0$.

It is possible to calculate more components in the decomposition series to enhance the approximation.

The resulting components using initial condition (2) are

$$
\begin{gathered}
u_{0}(x, t)=\frac{\alpha}{2} \operatorname{sech}^{2}\left(\frac{\sqrt{\alpha}}{2} x\right) \\
u_{1}(x, t)=L^{-1}\left(-6 A_{0}-u_{0 x x x}\right)=\frac{1}{2} \frac{\sinh (0.5 \sqrt{\alpha} x) \alpha^{5 / 2} t}{\cosh ^{3}(0.5 \sqrt{\alpha} x)} \\
u_{2}(x, t)=L^{-1}\left(-6 A_{1}-u_{1 x x x}\right)=\frac{1}{8} \frac{t^{2} \alpha^{4}\left(2 \cosh ^{2}(0.5 \sqrt{\alpha} x)-3\right)}{\cosh ^{4}(0.5 \sqrt{\alpha} x)} \\
u_{3}(x, t)=L^{-1}\left(-6 A_{2}-u_{2 x x x}\right)=\frac{1}{12} \frac{\sinh (0.5 \sqrt{\alpha} x) t^{3}\left(2 \cosh ^{2}(0.5 \sqrt{\alpha} x)-3\right) \alpha^{11 / 2}}{\cosh ^{5}(0.5 \sqrt{\alpha} x)}
\end{gathered}
$$


So, the solution in a series form is given by

$$
\begin{gathered}
u(x, t)=\frac{\alpha}{2} \operatorname{sech}^{2}\left(\frac{\sqrt{\alpha}}{2} x\right)+\frac{1}{2} \frac{\sinh (0.5 \sqrt{\alpha} x) \alpha^{5 / 2} t}{\cosh ^{3}(0.5 \sqrt{\alpha} x)}+\frac{1}{8} \frac{t^{2} \alpha^{4}\left(2 \cosh ^{2}(0.5 \sqrt{\alpha} x)-3\right)}{\cosh ^{4}(0.5 \sqrt{\alpha} x)} \\
+\frac{1}{12} \frac{\sinh (0.5 \sqrt{\alpha} x) t^{3}\left(2 \cosh ^{2}(0.5 \sqrt{\alpha} x)-3\right) \alpha^{11 / 2}}{\cosh ^{5}(0.5 \sqrt{\alpha} x)}
\end{gathered}
$$

And the resulting components using initial condition (6) are

$$
\begin{gathered}
u_{0}(x, t)=\frac{12(3+4 \cosh (2 x)+\cosh (4 x)}{(3 \cosh (x)+\cosh (3 x))^{2}} \\
u_{1}(x, t)=L^{-1}\left(-6 A_{0}-u_{0 x x x}\right)=\frac{48 t \sinh (x)\left(\cosh ^{2}(x)+6\right)}{\cosh ^{5}(x)} \\
u_{2}(x, t)=L^{-1}\left(-6 A_{1}-u_{1 x x x}\right)=\frac{96 t^{2}\left(2 \cosh ^{6}(x)+117 \cosh ^{4}(x)-96 \cosh ^{2}(x)-63\right)}{\cosh ^{8}(x)} \\
u_{3}(x, t)=L^{-1}\left(-6 A_{2}-u_{2 x x x}\right) \\
=\frac{256 t^{3} \sinh (\mathrm{x})\left(2 \cosh ^{8}(x)+1002 \cosh ^{6}(x)-594 \cosh ^{4}(x)-1728 \cosh ^{2}(x)-405\right)}{\cosh ^{11}(x)}
\end{gathered}
$$

So, the solution in a series form is given by

$$
\begin{gathered}
u(x, t)=\frac{12(3+4 \cosh (2 x)+\cosh (4 x)}{(3 \cosh (x)+\cosh (3 x))^{2}}+\frac{48 t \sinh (x)\left(\cosh ^{2}(x)+6\right)}{\cosh ^{5}(x)} \\
+\frac{96 t^{2}\left(2 \cosh ^{6}(x)+117 \cosh ^{4}(x)-96 \cosh ^{2}(x)-63\right)}{\cosh ^{8}(x)} \\
+\frac{256 t^{3} \sinh (\mathrm{x})\left(2 \cosh ^{8}(x)+1002 \cosh ^{6}(x)-594 \cosh ^{4}(x)-1728 \cosh ^{2}-1728 \cosh ^{2}(x)-405\right)}{\cosh ^{11}(x)}
\end{gathered}
$$

In order to prove numerically whether the application of padé approximants (Wazwaz, 1999; Mousa \& Kaltayev, 2009) to Adomian's series solution of KdV equation leads to better accuracy and larger convergence region, the numerical solution of examples with and without PA were evaluated. We concluded, from the considered examples, that PA improve the convergence region and the accuracy of the solution, except in the increasing in time. Also, we observe that the method of lines is better and higher accuracy.

We plot the profiles of the single soliton at $\alpha=1, \mathrm{t}=0,1,2,3$ using ADM and ADM padé approximation (ADMPA). 

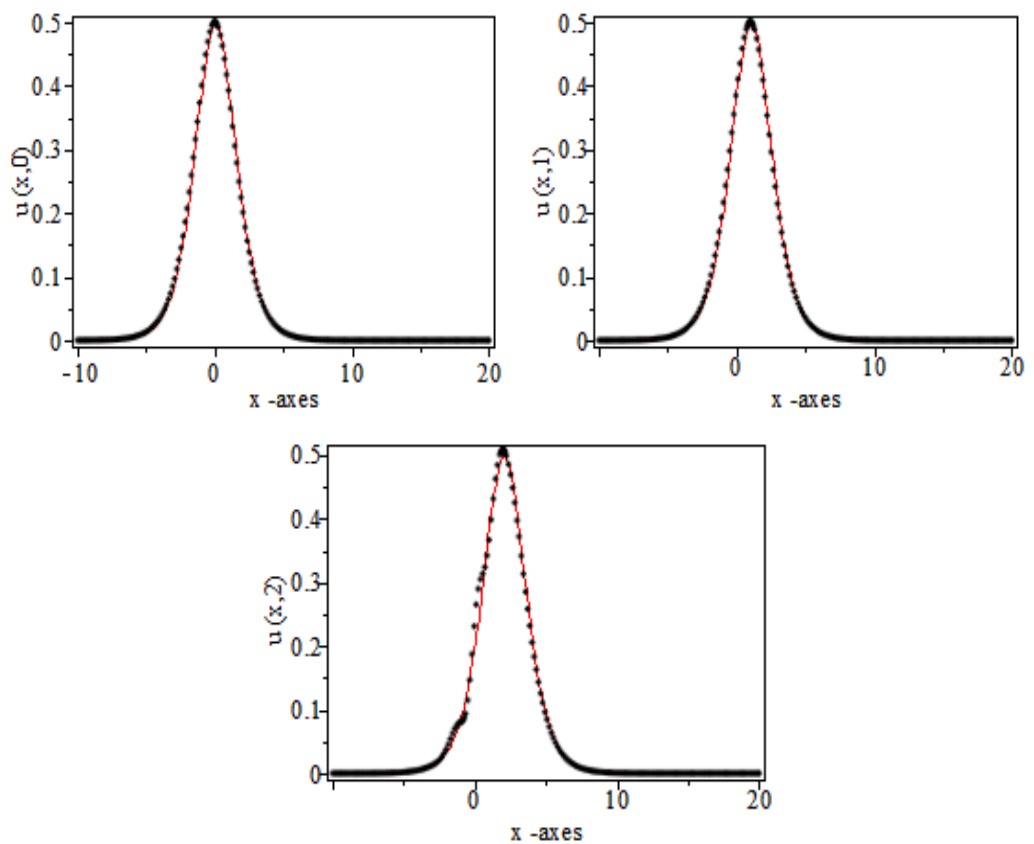

Figure 7. Comparison of ADM (dotted line) and exact (solid line) solutions corresponding to Example 1 at $\mathrm{t}=0$, 1,2

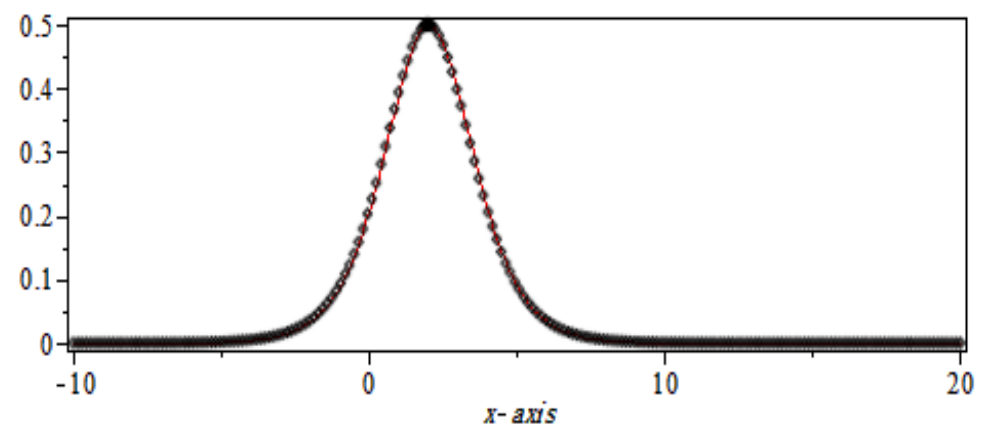

Figure 8. Comparison of the ADM padé approximation (dotted line) and exact (solid line) solutions corresponding to Example 1 at $\mathrm{t}=2$

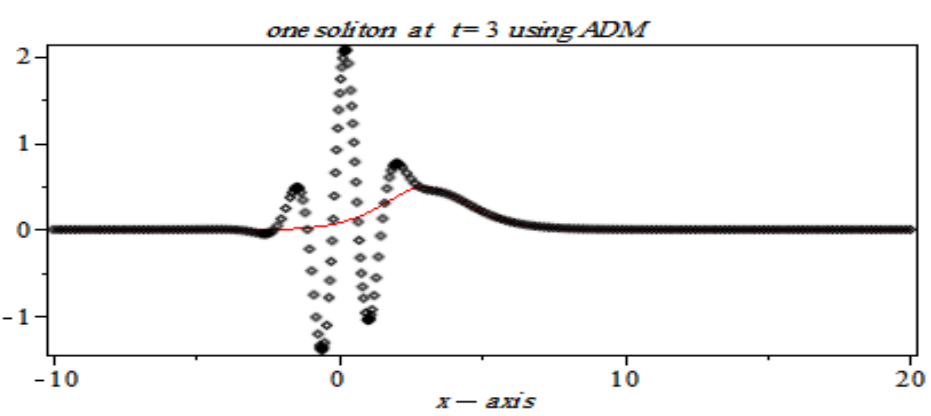

Figure 9. Comparison of the ADM (dotted line) and exact (solid line) solutions corresponding to Example 1 at $\mathrm{t}=3$ 


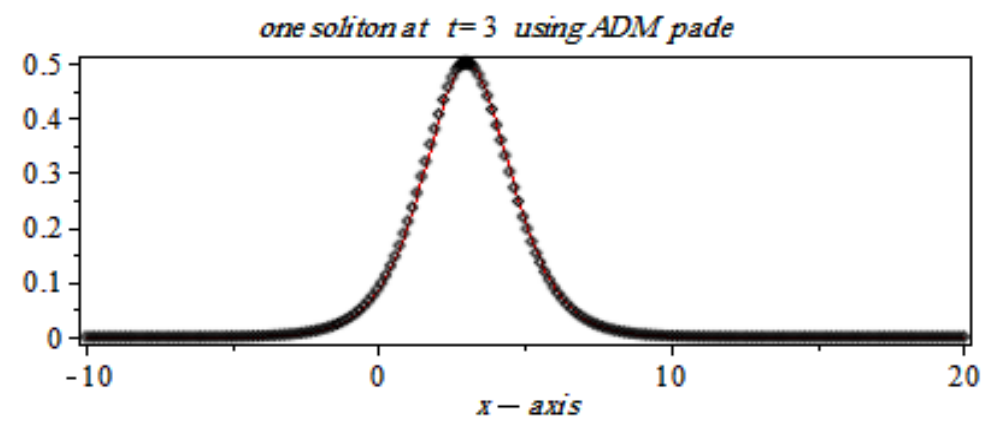

Figure 10. Comparison of the ADM padé approximation (dotted line) and exact (solid line) solutions corresponding to Example 1 at $\mathrm{t}=3$
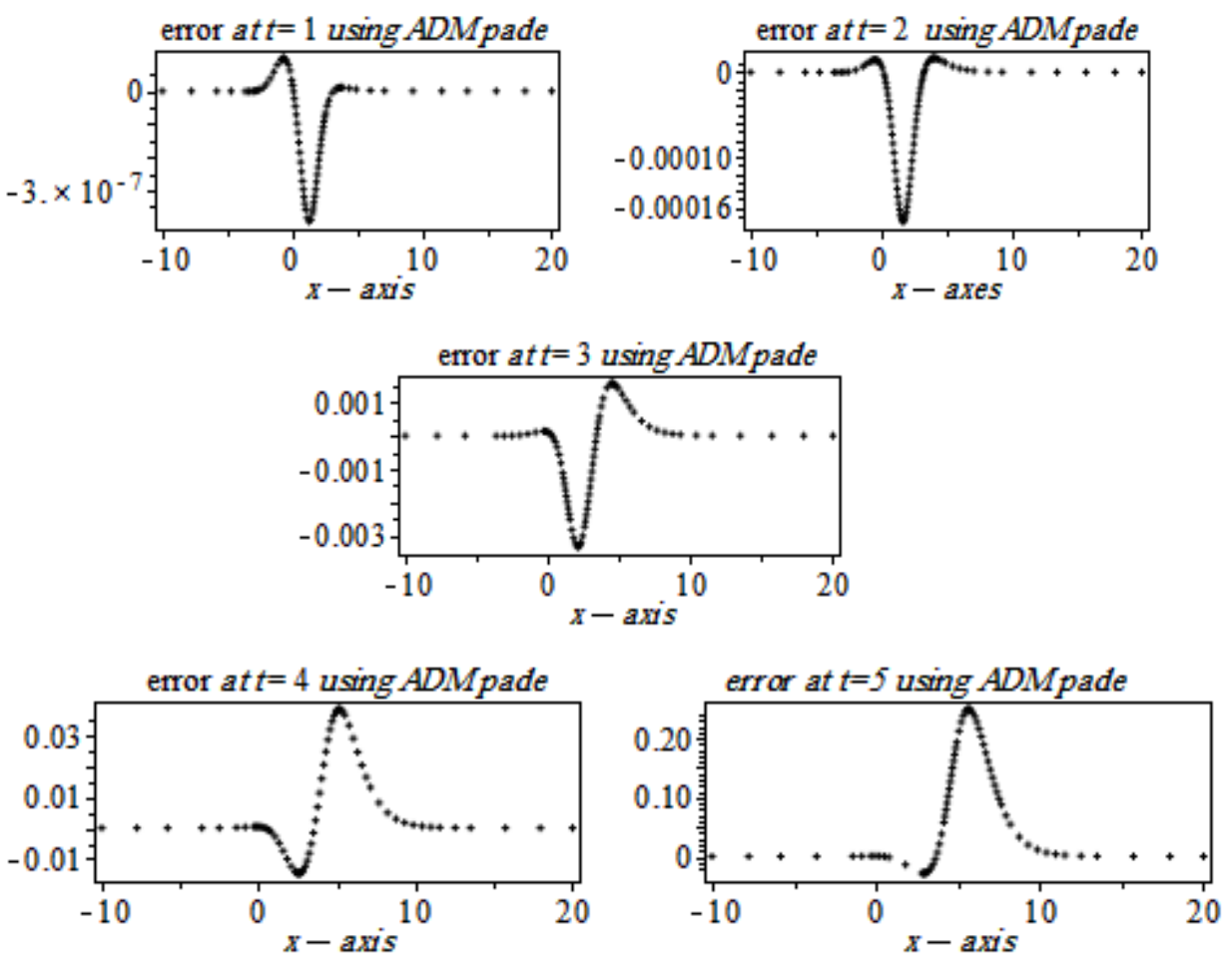

Figure 11. The error between the exact solution $u(\mathrm{x}, \mathrm{t})$ and the ADMPA at $t$ from 1 to 5

The profiles of the interacting of two solitons using ADM or ADMPA are ignored to be plotted because of the poor of the illustration due to the high error at high t. So, we only explained the obtained results though the following tables. 
Table 1. The absolute error of MOL I, MOL II and ADMPA approximation solutions for $\mathrm{N}=200, \alpha=1$, for example 1

\begin{tabular}{lllllll}
\hline $\mathrm{x}$ & $\begin{array}{l}\text { Error of } \\
\text { MOL I at } \\
\mathrm{t}=1\end{array}$ & $\begin{array}{l}\text { Error of } \\
\text { ADMPA at } \\
\mathrm{t}=1\end{array}$ & $\begin{array}{l}\text { Error of } \\
\text { MOL II at } \\
\mathrm{t}=1\end{array}$ & $\begin{array}{l}\text { Error of } \\
\text { MOL I at } \\
\mathrm{t}=3\end{array}$ & $\begin{array}{l}\text { Error of } \\
\text { ADMPA at } \\
\mathrm{t}=3\end{array}$ & $\begin{array}{l}\text { Error of } \\
\text { MOL II at } \\
\mathrm{t}=3\end{array}$ \\
\hline-8 & 0.00000655 & $8.124 * 10^{-9}$ & 0.0004 & $4.061 * 10^{-6}$ & $2.292^{*} 10^{-5}$ & 0.000981 \\
-6 & $2.406 * 10^{-5}$ & $1.267 * 10^{-7}$ & 0.001021 & $7.82 * 10^{-7}$ & $9.225^{*} 10^{-5}$ & $6.36^{*} 10^{-5}$ \\
-2 & $3.431 * 10^{-5}$ & $1.465 * 10^{-6}$ & $4.5 * 10^{-5}$ & $2.72 * 10^{-5}$ & 0.1950573 & 0.020999 \\
0 & $1.671 * 10^{-5}$ & $5.097 * 10^{-5}$ & 0.00257 & $2.291 * 10^{-5}$ & 1.4682495 & 0.0121929 \\
2 & $2.342 * 10^{-5}$ & $1.491 * 10^{-5}$ & 0.00128 & $2.483 * 10^{-5}$ & 0.048744 & 0.0110401 \\
4 & $1.254 * 10^{-5}$ & $2.642 * 10^{-5}$ & 0.00033 & $1.660^{*} 10^{-5}$ & 0.0087789 & 0.0186170 \\
8 & $6.571 * 10^{-6}$ & $2 * 10^{-5}$ & $4.94 * 10^{-5}$ & $2.071 * 10^{-5}$ & $3.27 * 10^{-6}$ & 0.0083711 \\
10 & 0.00001717 & $865^{*} * 10^{-5}$ & $3 * 10^{-5}$ & 0.000017387 & $4.023 * 10^{-7}$ & 0.0012240 \\
12 & $1.056^{*} 10^{-5}$ & $212 * 10^{-5}$ & $9.63 * 10^{-6}$ & $3.082 * 10^{-5}$ & $7.86^{*} * 10^{-6}$ & $1.6 * 10^{-5}$ \\
\hline
\end{tabular}

Table 2. The absolute error of MOL I, MOL II and ADMPA approximation solutions for $N=200, \alpha=4$ for example 1

\begin{tabular}{lllllll}
\hline $\mathrm{x}$ & $\begin{array}{c}\text { Error of } \\
\text { MOL I at } \\
\mathrm{t}=1\end{array}$ & $\begin{array}{c}\text { Error of } \\
\text { ADMPA at } \\
\mathrm{t}=1\end{array}$ & $\begin{array}{c}\text { Error of } \\
\text { MOL II at } \\
\mathrm{t}=1\end{array}$ & $\begin{array}{c}\text { Error of } \\
\text { MOL I at } \\
\mathrm{t}=2\end{array}$ & $\begin{array}{c}\text { Error of } \\
\text { ADMPA at } \\
\mathrm{t}=2\end{array}$ & $\begin{array}{c}\text { Errorof } \\
\text { MOL II at } \\
\mathrm{t}=2\end{array}$ \\
\hline 0 & $6.346^{*} 10^{-5}$ & 0.006123 & 0.00625 & 0.0002785 & 0.001264741 & 0.005986162 \\
1 & $2.955^{*} 10^{-5}$ & 0.03357 & 0.00309 & 0.00049 & 0.00158 & 0.007266771 \\
2 & 0.00103972 & 0.03561 & 0.024412 & 0.00054 & 0.00367 & 0.001421027 \\
3 & 0.00166716 & 0.799379 & 0.011636 & 0.000231 & 0.00028 & 0.000980707 \\
4 & 0.00244057 & 1.994413 & 0.00402 & 0.000242 & 0.002595 & 0.007517056 \\
5 & 0.00351941 & 0.839191 & 0.04951 & 0.00053 & 0.01972 & 0.007874936 \\
6 & 0.00030092 & 0.141199 & 0.00818 & 0.00124 & 0.1413 & 0.111189610 \\
7 & 0.00010097 & 0.019718 & 0.00039 & 0.00449 & 0.8444366 & 0.66482124 \\
8 & $2.024 * 10^{-5}$ & 0.00268 & 0.00328 & 0.00177 & 2.0017707 & 2.022917732 \\
9 & $2.028 * 10^{-5}$ & 0.000363 & 0.00311 & 0.006286 & 0.8336631 & 0.951241571 \\
\hline
\end{tabular}


Table 3. The absolute error of MOL I, MOL II and ADMPA approximation solutions for $N=500$ for example 2

\begin{tabular}{lllllll}
\hline & $\begin{array}{l}\text { Error of } \\
\mathrm{xOL} I \text { at }\end{array}$ & $\begin{array}{l}\text { Error of } \\
\text { ADMPA } \\
\mathrm{t}=0.1\end{array}$ & $\begin{array}{l}\text { Error of } \\
\text { MOL II at } \\
\mathrm{t}=0.1\end{array}$ & $\begin{array}{l}\text { Error of } \\
\text { MOL I at } \\
\mathrm{t}=0.2\end{array}$ & $\begin{array}{l}\text { Error of } \\
\text { ADMPA at } \\
\mathrm{t}=0.2\end{array}$ & $\begin{array}{l}\text { Error of } \\
\text { MOL II at } \\
\mathrm{t}=0.2\end{array}$ \\
\hline-7.5 & 0.00000297 & $1.8 * 10^{-8}$ & 0.00006755 & 0.0000082 & 0.00000008 & 0.00078998 \\
-5.1 & 0.00000366 & $1 * 10^{-8}$ & 0.00047041 & 0.0000168 & $8.01 * 10^{-7}$ & 0.00241833 \\
-3 & $3.47 * 10^{-6}$ & $3 * 10^{-6}$ & 0.0252515 & $1.181 * 10^{-5}$ & $8.240^{*} 10^{-5}$ & 0.01544084 \\
-1.2 & $2.277 * 10^{-5}$ & 0.004755 & 0.7659380 & $5.68 * 10^{-5}$ & 0.03905287 & 0.03839698 \\
0 & $1.017 * 10^{-5}$ & 2.440337 & 0.3277002 & $4.658^{*} 10^{-5}$ & 1.88919517 & 0.87788986 \\
3 & $7.3 * 10^{-7}$ & 0.047589 & 0.46572542 & 0.0006676 & 3.52226765 & 0.04972225 \\
6 & $1 * 10^{-8}$ & $1.2 * 10^{-7}$ & 0.00033343 & $1.186^{*} 10^{-7}$ & 0.00149757 & 0.00209393 \\
7.5 & $2 * 10^{-7}$ & 0.00031 & 0.00001457 & $1.178^{*} 10^{-6}$ & 0.00000295 & 0.00004445 \\
\hline
\end{tabular}

Table 4. The absolute error of MOL I, MOL II and ADMPA approximation solutions for $N=500$ for example 2

\begin{tabular}{lllllll}
\hline $\mathrm{x}$ & $\begin{array}{l}\text { Error of } \\
\text { MOL I at } \\
\mathrm{t}=0.4\end{array}$ & $\begin{array}{l}\text { Error of } \\
\text { ADMPA at } \\
\mathrm{t}=0.4\end{array}$ & $\begin{array}{l}\text { Error of } \\
\text { MOL II at } \\
\mathrm{t}=0.4\end{array}$ & $\begin{array}{l}\text { Error of } \\
\text { MOL I at } \\
\mathrm{t}=0.8\end{array}$ & $\begin{array}{l}\text { Error of } \\
\text { ADMPA at } \\
\mathrm{t}=0.8\end{array}$ & $\begin{array}{l}\text { Error of } \\
\text { MOL II at } \\
\mathrm{t}=0.8\end{array}$ \\
\hline-3 & $2.514 * 10^{-5}$ & 0.0003879 & 0.00968979 & $7.0147 * 10^{-5}$ & -0.00023 & 0.02048 \\
-1.2 & $7.75 * 10^{-6}$ & 0.02858793 & 0.08635220 & $2.3291 * 10^{-5}$ & -0.00079 & 0.01382 \\
0 & $3.2 * 10^{-7}$ & 0.77718936 & 0.73347406 & $7.2409 * 10^{-5}$ & 0.039457 & 0.008117 \\
1.8 & $4.165 * 10^{-5}$ & 1.11490356 & 0.02580017 & 0.00066959 & 1.038545 & 0.233115 \\
3 & $2.19 * 10^{-6}$ & 0.14668776 & 0.01652606 & 0.00092355 & 1.773964 & 0.23895 \\
6 & 0.0017078 & 1.89069863 & 0.07236627 & $6.8735^{*} 10^{-5}$ & 0.009807 & 0.00218 \\
7.5 & 0.0009451 & 1.09672645 & 0.01556362 & $3.5378^{*} 10^{-5}$ & 0.000489 & 0.00323 \\
9 & $4.2 * 10^{-7}$ & 0.00291482 & 0.00334152 & $6.4837 * 10^{-5}$ & 0.000027 & 0.00084 \\
\hline
\end{tabular}

From the above tables we can infer that ADM have a better convergence. However, a closer look at the errors of $\mathrm{ADM}$ reveals that the error considerably increments in the columns as $t$ increase. This is an indication of little stability on the part of ADM, in contrast to the MOL.

\section{Conclusion}

In this article, the method of lines and Adomian decomposition method have been implemented for obtaining solitary wave solutions of the KdV equations. The results show that the considered methods are powerful mathematical tools for obtaining accurate solutions such equation. A comparison between MOL and ADM shows that the accuracy of MOL is better than that in the ADM for both one and two solitons solutions. Moreover, MOL can overcome difficulties arising in the calculation of Adomian's polynomials. Therefore the MOL is much easier and more convenient to apply than ADM. The computations associated with examples provided here were performed using Maple 15.

\section{References}

Adomian, G. (1994). Solving Frontier Problems of Physics: The Decomposition Method. Boston, MA: Kluwer Academic Publishers.

Ali, A. H. A. (1989). Finite element studies of the Korteweg-de Vries equation ( $\mathrm{PhD}$ thesis). University of Wales, UK.

Baker, G. A. (1975). Essentials of padé approximants. New York: Academic Press.

Baker, G. A. (1996). Graves-Morris, P. padé approximants. New York: Cambridge University Press. http://dx.doi.org/10.1017/CBO9780511530074 
Debnath, L. (1994). Nonlinear water waves. Boston, MA: Academic press.

Debnath, L. (1998). Nonlinear partial differential equations and engineers. Berlin: Birkhauser.

Drazin, P. G. (1989). Johnson RS. Solitons: an introduction. Cambridge: Cambridge University Press. http://dx.doi.org/10.1017/CBO9781139172059

Gardner, L. R. T., \& Gardner, G. A. (1990). Solitary waves of the regularized long wave equation. J. Comput. Phys, 91, 441-459. http://dx.doi.org/10.1016/0021-9991(90)90047-5

Gardner, L. R. T., \& Gardner, G. A. (1992). Solitary waves of the equal width wave equation. J. Comput. Phys, 101(1), 218-223. http://dx.doi.org/10.1016/0021-9991(92)90054-3

Hirota R. (1973b). Exact N-soliton solutions of the wave equation of long waves in shallow-water and in nonlinear lattices. J. Math. Phys., 7, 810-814. http://dx.doi.org/10.1063/1.1666400

Hirota, R. (1973a). Exact N-soliton solutions of a nonlinear wave. J. Math. Phys., 14(7), 805-809. http://dx.doi.org/10.1063/1.1666399

Kening, C. E., Ponce, G., \& Vega, L. (1997). Global solutions for the KdV equation with unbounded data. $J$ Differential Equations, 139, 339-64. http://dx.doi.org/10.1006/jdeq.1997.3297

Khattak, A. J., \& Siraj-ul-Islam. (2008). A comparative study of numerical solutions of a class of KdV equation. Appl. Math. Comput., 199, 425-434. http://dx.doi.org/10.1016/j.amc.2007.10.002

Korteweg, D. J., \& de Vries, G. (1895). On the change of form of long waves Advancing in rectangular canal, and on a new type of long stationary wave. Philos. Mag, 39, 422-443.

Lax, P. D. (1978). A Hamiltonian approach to the KdV and other equations. In Crandall M. (Ed.), Nonlinear evolution equations $(11,207-224)$. New York: Academic press.

Luwai, W. (2009). A modified tanh-coth method for solving the KdV and the KdV-Burgers equations. Journal of Communication in Nonlinear Science and Numerical Simulation, 14(2), 443-450.

Mehdi, D. (2004). Application of the Adomian decomposition method for two-dimensional parabolic equation subject to nonstandard boundary specifications. Appl. Math. Comput, 157, 549-560. http://dx.doi.org/10.1016/j.amc.2003.08.098

Mousa, M. M., \& Kaltayev, A. (2009). The Homotopy perturbation padé technique for constructing approximate and exact solutions of Boussinesq equations. Applied Mathematical Sciences, 3(22), 1061-1069.

Mousa, M. M., Ragab Shahwar, F., \& Mostafa, S. I. (2006). New exact solutions for nonlinear equations by the general improved Tanh and Sech methods. Sci. Bull. Fac. Eng. Ain shams Univ. Egypt, 41(2), 859-876.

Ozis, T., \& Ozer, S. (2006). A simple similarity-transformation-iterative scheme applied to Korteweg-de Vries equation. Applied Mathematical Sciences, 173(1), 19-32.

Pregla, R., \& Ahlers, E. (1993). Method of lines for analysis of discontinuities in optical waveguides. Electronics Letters, 29, 1845-1851. http://dx.doi.org/10.1049/el:19931228

Pregla, R., \& Yang, W. (1993). Method of lines for analysis of multilayered dielectric waveguides with bragg gratings. Electronics Letters, 29, 1962-1957. http://dx.doi.org/10.1049/el:19931306

Rogge, U., \& Pregla, R. (1991). Method of lines for the analysis of strip-loaded optical waveguides. Optical Society of America (B), 8, 459-463. http://dx.doi.org/10.1364/JOSAB.8.000459

Schiesser, W. E. (1991). The numerical method of lines: integration of partial differential equations. San Diego, California: Academic Press. http://dx.doi.org/10.1049/el:19950636

Wazwaz, A. M. (1999). The modified decomposition method and padé approximants for solving the Thomas-Fermi equation. Appl. Math. Comput, 105, 11-19. http://dx.doi.org/10.1016/S0096-3003(98)10090-5

Wazwaz, A. M. (1999). A new algorithm for calculating Adomian polynomial for nonlinear operators. Appl. Math. Comput, 111, 53-69.

Wazwaz, A. M. (2001). The numerical solution of fifth-order boundary value problems by the decomposition method. J. Comput. Appl.Math, 136, 259-270. http://dx.doi.org/10.1016/S0377-0427(00)00618-X

Yang, W. D., \& Pregla, R. (1995). Method of lines for analysis of waveguide structures with multi discontinuities. Electronics Letters, 31, 892-893. 
Zabusky, N. J., \& Kruskal, M. D. (1965). Interaction of Solitons in Collision less Plasma and Recurrence of Initial States. Phys. Rev. Lett., 6, 240-243. http://dx.doi.org/10.1103/PhysRevLett.15.240 